journal club

\title{
Dabigatran bewährt sich in der Praxis
}

Fragestellung: Wie ist die Wirksamkeit und Sicherheit von Dabigatran zur Schlaganfall-Prävention bei Patienten mit Vorhofflimmern im klinischen Alltag?

Hintergrund: Das orale Antikoagulans Dabigatran wurde in zwei Dosierungen in der RELY-Studie mit Warfarin verglichen und zeigte dort für die niedrige Dosis Nichtunterlegenheit und für die höhere Dosis Überlegenheit bezüglich des Endpunktes Schlaganfall und systemische Embolie. Patienten in großen randomisierten Studien sind allerdings nicht immer mit den Patienten im klinischen Alltag vergleichbar. Daher ist es sehr wichtig, Behandlungsdaten und unerwünschte Ereignisse entweder aus großen Registern oder populationsbezogenen Datenbanken zu erhalten.

Patienten und Methodik: In Dänemark gibt es drei Register die gut validiert sind: Im nationalen Zivilregister sind die biologischen Daten von allem in Dänemark geborener Menschen erfasst. Das dänische nationale Patientenregister, das seit 1977 besteht, erfasst bei allen Menschen, die in Dänemark in ein Krankenhaus eingewiesen werden, die Entlassungsdiagnose und das Datum der Krankenhausaufnahme. Seit 1994 werden in Dänemark alle Verschreibungen für Medikamente in einem zentralen Register erfasst. Da jeder dänische Staatsbürger eine Identifikationsnummer hat, können die Daten zwischen den drei Registern abgeglichen und ausgewertet werden. Im Rahmen der Studie wurden alle Patienten erfasst, die nach dem 1. August 2011 mit einer der beiden Dosierungen von Dabigatran oder Warfarin aufgrund von Vorhofflimmern behandelt wurden. Die Auswertung geht bis zum 31. Dezember 2012. Erfasst wurden allerdings nur Patienten, die zum ersten Mal eine Verschreibung für ein Antikoagulans erhielten. Die primären Endpunkte der Studie waren Schlaganfall, systemische Embolien, intrakranielle Blutung. Sekundäre Endpunkte waren Tod, gastrointestinale Blutungen, traumatische intrakranielle Blutungen oder schwerwiegende Blutungen. Außerdem wurden Myokardinfarkte, Lungenembolien und Krankenhausaufnahmen erfasst. Die Auswertung selbst erfolgte mit Hilfe von Propensity Scores. Hierbei werden für jeden Patienten der Dabigatran erhält, zwei Patienten ausgewählt, die mit Warfarin behandelt wurden und die ein vergleichbares Risikoprofil aufweisen. Die wichtigsten Faktoren die korrigiert wurden, waren Alter und Geschlecht.

Larsen TB, Rasmussen LH, Skjøth $\mathrm{F}$ et al. Efficacy and safety of dabigatran etexilate and warfarin in ,real world' patients with atrial fibrillation: A prospective nationwide cohort study. J Am Coll Cardiol 2013; 61: 2264-73 koreduktion von $27 \%$, für die hohe Dosis eine Risikoerhöhung von $18 \%$. Beide Werte waren statistisch nicht signifikant. Intrakranielle Blutungen waren für beide Dosierungen von Dabigatran signifikant erniedrigt gegenüber Warfarin, mit einer Risikoreduktion von $76 \%$ für die niedrige Dosis und $92 \%$ für die höhere Dosis. Die Mortalität war für beide Dabigatran-Dosierungen signifikant reduziert. Auch Myokardinfarkte waren signifikant seltener, mit einer Risikoreduktion von $70 \%$ für die niedrige Dosis und $60 \%$ für die hohe Dosis von Dabigatran. Gastrointestinale Blutungen waren um $40 \%$ geringer unter der niedrigen Dosis von Dabigatran verglichen mit Warfarin. Für die höhere Dosis bestand eine Risikoerhöhung von $12 \%$ die statistisch nicht signifikant war. Krankenhausaufnahmen waren für beide Dosierungen von Dabigatran signifikant seltener als unter Warfarin. Schwerwiegende Blutungskomplikationen waren insgesamt nummerisch unter Dabigatran geringer, der Unterschied war hier allerdings statistisch nicht signifikant.

Schlussfolgerungen: In dieser Registeranalyse von Patienten mit Vorhofflimmern waren beide Dosierungen von Dabigatran zur Schlaganfall-Prävention genauso wirksam wie Warfarin. Es ergaben sich signifikant niedrigere Raten für Sterblichkeit, intrakranielle Blutungen, Lungenembolien und Myokardinfarkte unter Dabigatran gegenüber Warfarin.

\section{- Kommentar von Hans-Christoph Diener, Essen Beruhigende Ergebnisse}

Die Ergebnisse dieser großen populationsbezogenen Studie sind sehr beruhigend, da sie in weiten Teilen die Ergebnisse der RELY-Studie widerspiegeln. Für Neurologen relevant ist die dramatische Reduktion von intrakraniellen Blutungen. Ein weiteres überraschendes Ergebnis ist die über 50\%ige Reduktion von Myokardinfarkten. Hier hatte sich bei einer relativ geringen Zahl von Ereignissen in der RELY-Studie ein gegenteiliger Trend ergeben. Daraufhin wurden eine Reihe von Metaanalysen durchgeführt die postulierten, dass Dabigatran möglicherweise Myokardinfarkte bedingen könnte. Dies ist nach den Ergebnissen des dänischen Registers sehr unwahrscheinlich. Es muss hier besonderes berücksichtigt werden, dass die Patienten bezüglich ihres Risikos für arterielle vaskuläre Ereignisse gematcht waren, was in den Studien zur Prävention tiefer Beinvenenthrombosen mit Dabigatran nicht notwendigerweise der Fall war. Die hier gewonnenen Daten passen auch gut zu den Ergebnissen eines großen Registers der amerikanischen Zulassungsbehörde, dass ebenfalls zeigt, dass das Risiko von intrakraniellen Blutungen unter Dabigatran signifikant erniedrigt ist. Im amerikanischen Register zeigte sich auch eine signifikante Reduktion von gastrointestinalen Blutungen unter der hohen Dosis von Dabigatran verglichen mit Warfarin. 